\title{
A Fuzzy Logic based Unequal Clustering Protocol for Heterogeneous Wireless Sensor Networks
}

\author{
Priya Bhatnagar \\ M.Tech, Dept. of Computer Science and \\ Engineering \\ Rajasthan College of Engineering for Women, \\ Jaipur, Rajasthan, India
}

\author{
Subhash Chandra \\ Assistant Professor, Dept. of Computer Science \\ and Engineering \\ Rajasthan College of Engineering for Women, \\ Jaipur, Rajasthan, India
}

\begin{abstract}
Wireless Sensor Networks (WSNs) have gained due importance for applications requiring remote sensing. The major challenges faced are related to energy and routing. The sensor nodes being battery operated devices of limited resources, techniques like data aggregation, selective node activation and energy efficient routing are adopted with main focus of saving energy. The objective is to prolong life of the network and also distribute the load evenly among the nodes. Several approaches have been suggested in literature; mostly for homogeneous WSNs only. Fuzzy logic has recently been opted for selection of cluster heads in these networks. This paper proposes a fuzzy logic based unequal clustering protocols for WSNs in heterogeneous settings. Four decision criteria for selecting cluster heads are input to the fuzzy logic which gives two outputs instead of one. This unique feature makes the proposed method effective.
\end{abstract}

\section{Keywords}

Wireless Sensor Networks, Cluster Heads, Network Lifetime, Energy Consumption, Heterogeneous Networks

\section{INTRODUCTION}

Over the years, Wireless Sensor Networks (WSNs) have been applied to fields like healthcare, military and defense services, environment sensing, infrastructure monitoring, event security and more, owing to their applicability and efficiency in various sensing and monitoring tasks [1]. To define, these networks consist of autonomous sensor nodes deployed in the Region-of-Interest (RoI) for sensing the area's physical and environmental conditions like pressure, temperature, sound and more. The sensing activity takes place from the source to sink (base station) in three phases - sensing, processing and transfer. All sensor nodes individually perform sensing of the interested RoI, process the sensed data using local processing tools and transfer the processed data to the desired location. WSNs, though efficient, undergo performance downfalls because of several issues [2]. The issues encountered are those of bandwidth, energy, memory and processing capabilities, out of which the energy inefficient routing of these networks is the most stressed upon topic. These nodes, being battery operated devices, consume a considerable amount of energy thereby affecting the total network lifetime. The matter is of much concern due to the irreplaceable nature of these batteries.

A promising alternative is to select some nodes of all as Cluster Heads (CHs) responsible for the aggregation and compression of the sensed data from its member nodes and transferring of the same to the BS. The positions of $\mathrm{CHs}$ are randomly rotated so as to not drain their entire energy. $\mathrm{CH}$ election might depend on different criteria, varying from protocol to protocol.

A recently adopted approach for $\mathrm{CH}$ election is Fuzzy logic[3][5]. Fuzzy logic uses Fuzzy if-then rules to convert fuzzy input linguistic variables into fuzzy output linguistic variables. The obtained fuzzy output variables are converted into a crisp value using a defuzzification operator. The output variables deduce the chances of a node to be selected as a $\mathrm{CH}$. Fuzzy Logic makes decision making related to the selection of $\mathrm{CH}$ and routing easier.

Though homogeneous WSNs have received much attention by researchers, there are many real life applications where heterogeneous deployment is present. Since the primary objective of any energy efficient protocol for WSNs should be to uniformly distribute load throughout the network, the problem in heterogeneous networks is much harder than that in homogeneous network where the node begins with the same energy level. We have proposed a protocol for heterogeneous WSNs. The proposed method uses fuzzy logic to incorporate as many factors that affect the decision of electing a $\mathrm{CH}$.

The paper can be organized as follows. Section II describes the proposed clustering method. Section III describes the simulation setup. The simulation results comparing the proposal and a recent work by Baranidharan and Santhi are listed in Section IV. Section V lists the contributions in the proposal and the future directions of work.

\section{PROPOSED CLUSTERING}

This section discusses the proposed method of cluster head selection, the criteria of selection and its frequency.

\subsection{Development of Idea}

Several research works agree that the degree of a node in the Wireless Sensor Network (WSN) should play a role in deciding whether a node should be elected as a Cluster Head $(\mathrm{CH})$ or not. While the degree is simply the number of neighbours of a node, certain new terms need to be introduced for heterogeneous settings. In a heterogeneous network, every node has different communication range. The nodes to which a certain node $\mathrm{x}$ can send messages are different from the nodes from which $\mathrm{x}$ receives messages. Hence, degree should be replaced by separate terms namely 'Indegree' and 'Outdegree' to denote the different numbers of nodes in respective category. This means new criteria and rules for decision of $\mathrm{CH}$ election need to be designed in heterogeneous WSNs. We propose a method based on fuzzy logic which treats indegree and outdegree as separate inputs. The 
following sections discuss the various aspects of the proposed $\mathrm{CH}$ election protocol.

\subsection{Assumptions}

The proposed UCFAH (Unequal Clustering using Fuzzy logic Applied to Heterogeneous wireless sensor networks) protocol follows the given assumptions.

- The sensor nodes in the underlying network are heterogeneous in nature.

- The nodes remain static after having been deployed in the Region of Interest (RoI).

- The distance between individual nodes is calculated through Received Signal Strength Indicator (RSSI).

- The distance to the base station of each individual node and its neighbours are calculated based on the message interactions between the sensor nodes and the base station. After deployment, a 'HELLO' message is sent from the base station to all the nodes. The nodes then send a 'HELLO' message to all nodes within its communication radius ' $\mathrm{R}$ '.

- $\quad$ A node dies only when its energy is over.

- Base station or the sink of the network has considerable network knowledge.

\subsection{Input and Output Parameters}

For selection of $\mathrm{CH}$ in the proposed protocol, four input parameters - Residual Energy, Outdegree, Indegree and Distance to the BS. Since the CHs have more responsibilities than the member nodes, remaining/residual energy of nodes is a powerful factor in selection. The number of nodes within the communication radius of a node is called its outdegree implying the nodes to which this node can send messages directly. A higher outdegree implies larger number of messages to be broadcasted by the node leading to more energy consumption and therefore a lesser chance of being elected as a $\mathrm{CH}$. The number of nodes from which a node can receive messages is called its indegree. A higher indegree is in favour of a node being selected as a $\mathrm{CH}$ and also will allow the $\mathrm{CH}$ to have many cluster members. Yet, a node should have a non-zero outdegree along with a high value of indegree. Nodes closer to the BS have fewer members to join them since the incoming traffic from distant nodes is already more. The nodes distant to BS have more member nodes but need more energy to forward its aggregated data.

The proposed protocol has two output variables instead of one - Chance(probability of a node of being elected as a $\mathrm{CH}$ ) and size(cluster size depending upon the indegree, outdegree and location of the $\mathrm{CH}$ )

\subsection{Phases}

The proposed protocol works in two phases - cluster formation and data collection. Fig. 1 gives the operational diagram of the proposed UCFAH protocol. The partitions in the data collection phase of the protocol are frames representing particular data collection rounds. In particular, it refers to the time of transmitting data between member nodes and $\mathrm{CH}$ nodes.

\subsubsection{Cluster Formation Phase}

The cluster formation is subdivided into $\mathrm{CH}$ election and Cluster Building phases. Selection of $\mathrm{CHs}$ is done based on fuzzy if-then rules

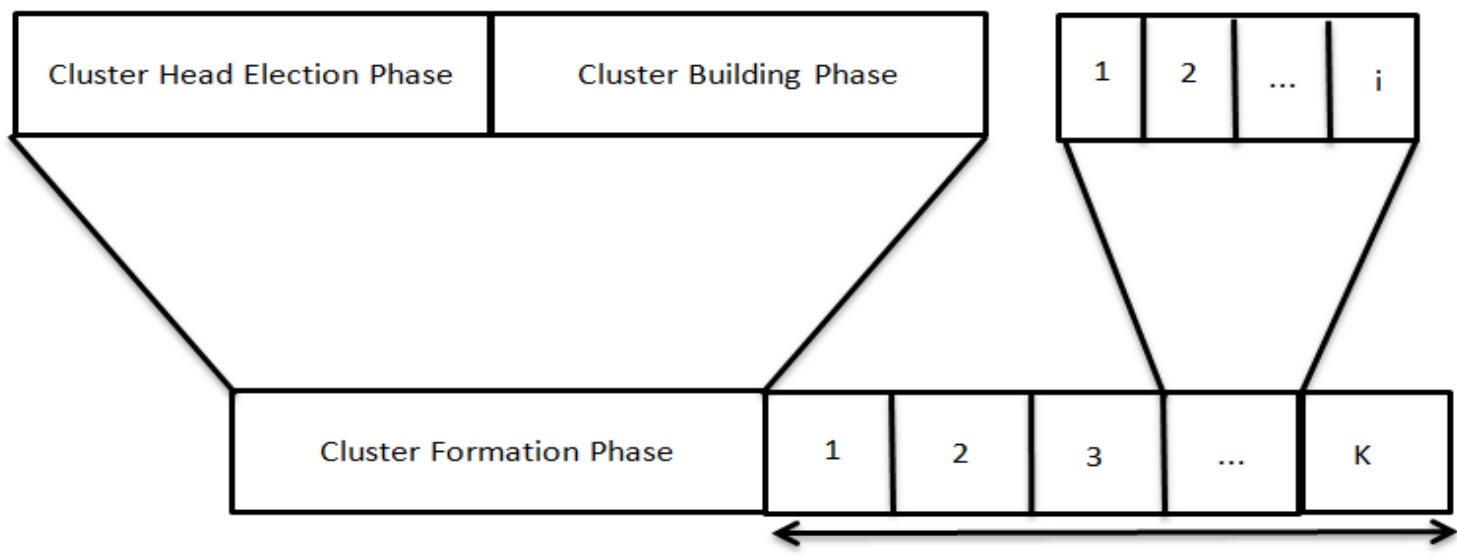

Data Aggregation Phase

Fig 1 Phases of the proposed protocol

The $\mathrm{CH}$ election phase starts by taking each node as the probationary $\mathrm{CH}$ nodes. The input and output variables take equivalent fuzzy linguistic variables - three each for all input variables, nine for output variable 'chance' and seven for output variable 'size'. All the fuzzy linguistic variables follow triangular membership function. Membership functions of the variables are depicted in Fig. 2. The values of input and output membership functions for fuzzification are picked from the description mentioned in [14]. While this applies to 'residual energy', 'distance to BS', 'chance' and 'size'; the values for 'outdegree' are chosen near to the values of variable 'degree' in [14]. Values of 'indegree' have been set through experimental analysis. Fuzzification of the crisp input values to suitable linguistic variables is done using Fuzzy Inference System (FIS) based on the provided membership functions. Development of the fuzzy if-then rules is done using Mamdani method [15] owing to the simplicity and effectiveness of the method. With four input variables having three fuzzy levels each, we have $3^{4}=81$ rules. The output to the FIS is also a fuzzy linguistic variable that is defuzzified to a crisp value using the Centre of Area method. The fuzzy rules are not listed due to page limitations.

On computing the chance, all nodes broadcast a 'CH.CANDIDATE' message to all nodes lying within the 
nodes' transmission radius ' $R$ '. The message contains node information and its computed 'chance'. Nodes, on comparing the 'chance' values of other nodes from one's own and finding no competition, elect themselves as $\mathrm{CHs}$ and broadcast a $\mathrm{CH}$ _WON message within the communication radius ' $R$ '. Each node may receive more than a single CH_WON message from its neighbours and joins the nearest $\mathrm{CH}$ of all by sending the CM_JOIN message. On receiving the $\mathrm{CH}_{-}$WON message from only one $\mathrm{CH}$, the node joins it by sending the CM_JOIN message. The CHs on receiving the CM_JOIN messages check for its capability to accept or reject new members based on its 'size' and accept the nodes if scope
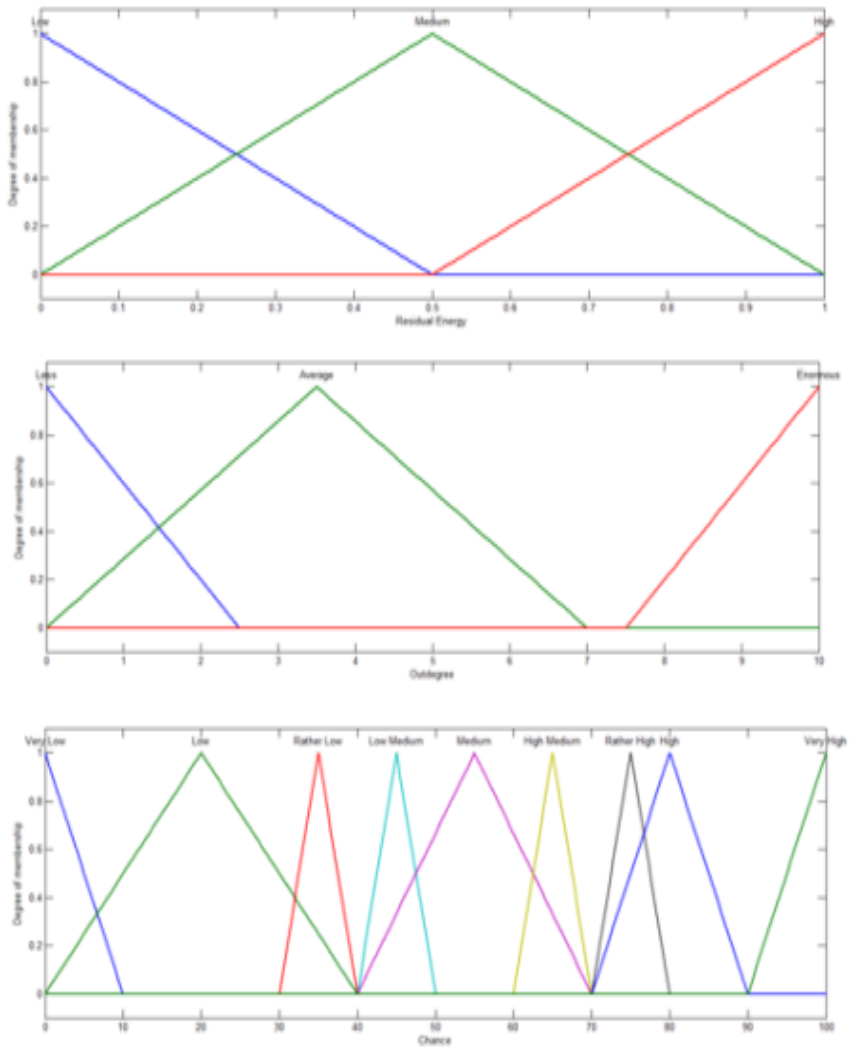

is there, that is if number of already accepted member nodes are less than the 'size' value. The $\mathrm{CHs}$ send a CM_ACCEPTANCE or a CM_REJECTION message to the nodes interested in joining accordingly. On receiving the CM_REJECTION message, the member node sends CM_JOIN to other nearest $\mathrm{CHs}$ except the $\mathrm{CH}$ that sent the CM REJECTION message. The process is repeated till it joins any of the CHs. A worst case scenario may also occur, when a member node cannot join any $\mathrm{CH}$ and therefore elects itself as $\mathrm{CH}$ so as to involve each node of the network in the cluster framework.
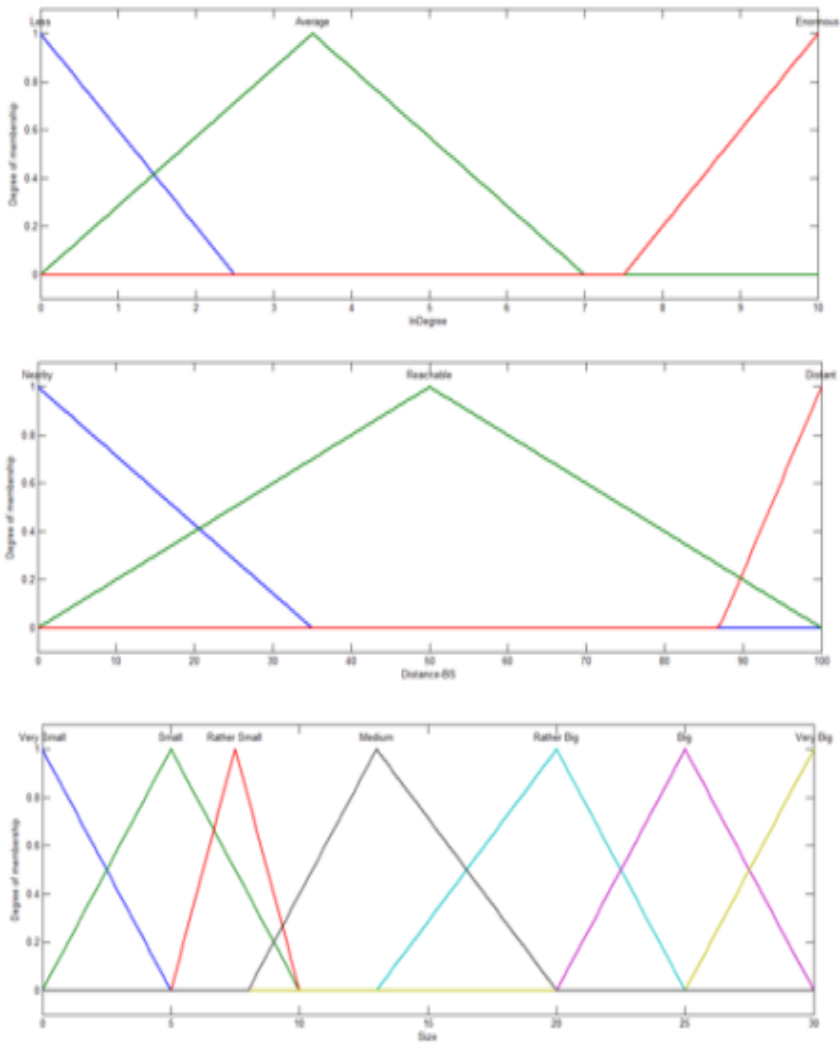

Fig. 2 Membership Functions

\subsubsection{Data Collection Phase}

On creation of clusters, a TDMA schedule is generated by each $\mathrm{CH}$ for its member node. Following the schedule, transmission of data from the member node to the $\mathrm{CH}$ is done after sensing. The member nodes will function only at the allotted time and are in a sleeping mode otherwise. The same is not possible with $\mathrm{CHs}$ because of being at the receiving end to incoming sensed data. The length of each frame is dependent on the size of the cluster and the time allotted to each member node for data transmission. The time allotted is same for each node and one data per frame is sent by each member. Aggregation of the data into a single packet is done by the $\mathrm{CHs}$ to send it to BS in a multi-hop manner. In order to prevent energy from wastage in terms of frequent clustering or other management tasks, more time is allotted in the data collection phase than the cluster formation phase.

\section{STATES}

Each node at the execution time of the proposed protocol transforms into any of the following four states - initial, probationary, final $\mathrm{CH}$ or member states. At the time of deployment, each node is in its initial state. The nodes with higher values of 'chance' enter the probationary $\mathrm{CH}$ node. If these nodes find no competition within their transmission radius ' $\mathrm{R}$ ' at the time of $\mathrm{CH}$ election phase, the nodes enter the final $\mathrm{CH}$ state or else the member state. At the start of the next clustering process, all nodes begin with the initial state. Fig. 8 shows the state transformation of a node.

\section{SIMULATION SETUP}

\subsection{Performance Measures}

The performance of the proposed and DUCF algorithms is compared for the total number of rounds executed, First Node Die, Half Node Die and Average Energy Consumed per Round.

\subsection{Simulation Parameters}

The simulation parameters, their descriptions and values are listed in Table 4.1. Initial energy of nodes is between $0.5 \mathrm{~J}$ and $1 \mathrm{~J}$. The threshold distance ' $\mathrm{d}_{\mathrm{o}}$ ' is fixed at $87 \mathrm{~m}$. The transmission and reception energy models are same like in [11 of DUCF].

$$
E_{\text {tx }}=l * E_{\text {elec }}+\varepsilon_{m p} * d^{4}, d>d_{0}
$$




$$
E_{t x}=l * E_{\text {elec }}+\varepsilon_{m p} * d^{2}, d<d_{0}
$$

And

$$
E_{r x}=l * E_{\text {elec }}
$$

The maximum communication range is $30 \mathrm{~m}$. The network is considered dead when $75 \%$ of the nodes die.
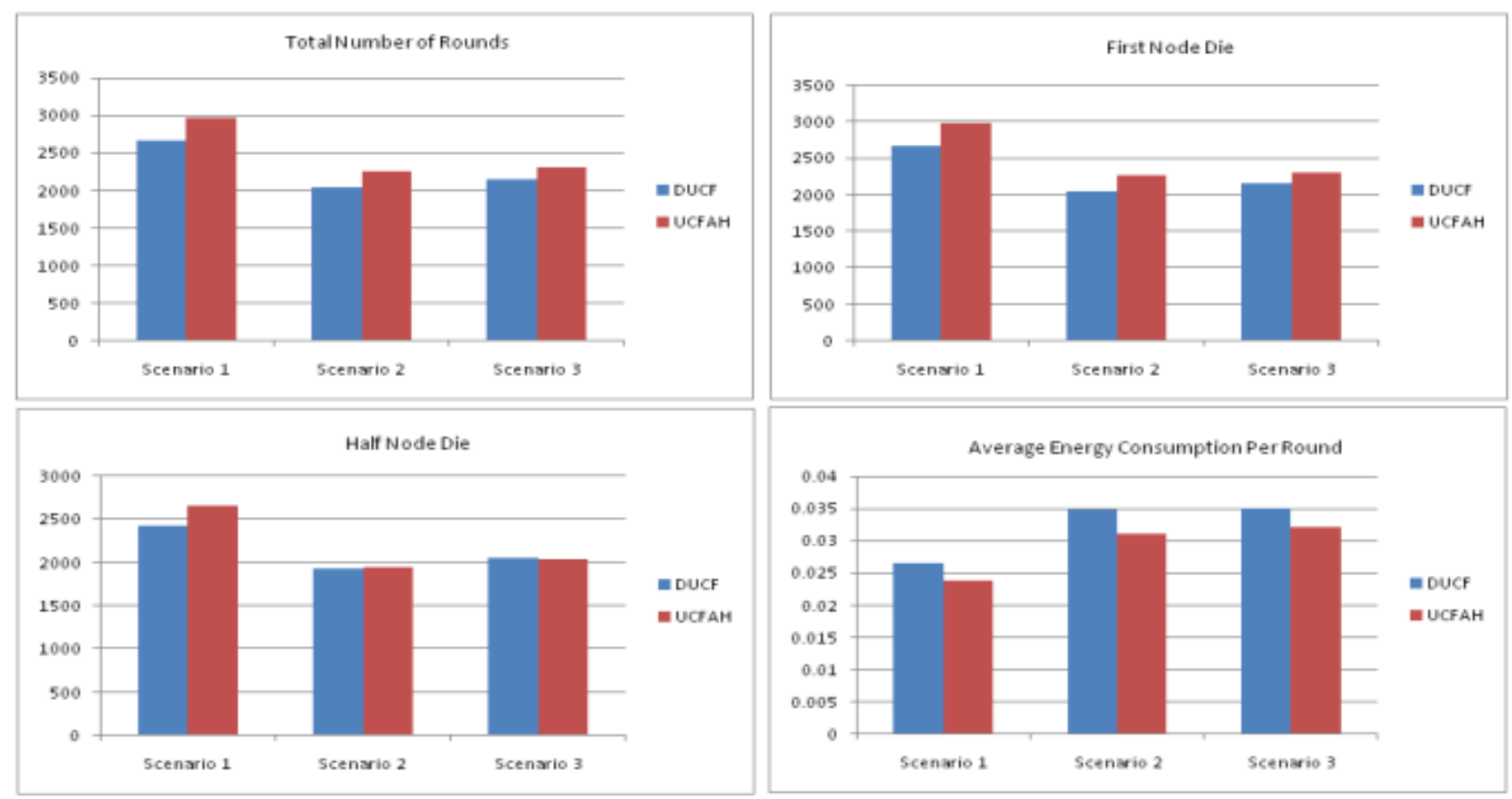

Figs. 3 Result of three scenario

\section{SIMULATION RESULTS}

The performance of the two algorithms - proposed and DUCF [14] is compared by varying the location of the BS and observing the values for the total number of rounds, FND, HND and AECR. The results of each for the three scenarios are depicted graphically in Fig. 3.

Figs. 4.1, 4.2 and 4.3 show the fall of total energy of the network as the protocol progresses for all three scenarios. It can be easily noted that the fall is slower for the proposed protocol as compared to DUCF.

\subsection{Scenarios Tested}

The proposed UCFAH and DUCF [14] protocol are compared on the basis of the discussed measures for three scenarios.

- Scenario 1: Base Station is within the RoI

- Scenario 2: Base Station is in the middle of RoI

- Scenario 3: Base Station is outside of Rol
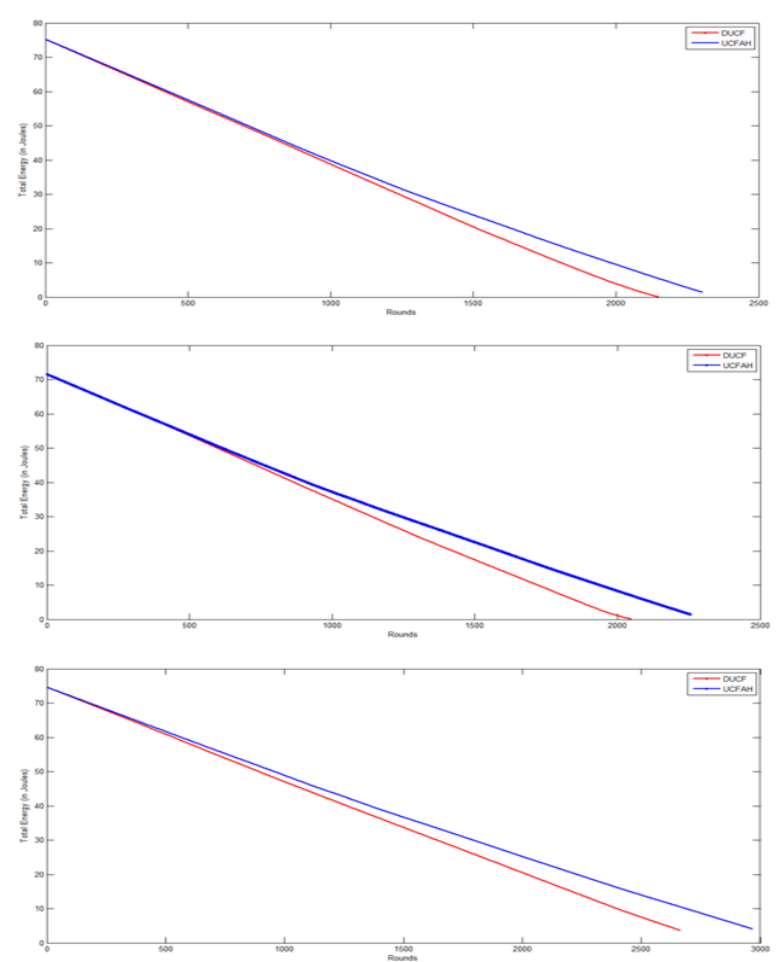

Figs. 4.1, 4.2 and 4.3 the fall of total energy for the proposed protocol and DUCF 
Table 4.1 Simulation parameters, Description and Values

\begin{tabular}{|c|l|l|}
\hline Parameter & Description & Values \\
\hline$l$ & $\begin{array}{l}\text { Number of bits to be } \\
\text { transmitted }\end{array}$ & 4000 \\
\hline$E_{\text {elec }}$ & $\begin{array}{l}\text { Energy spent in } \\
\text { transmission and reception } \\
\text { tasks }\end{array}$ & $50 \mathrm{~nJ} / \mathrm{bit}$ \\
\hline$\varepsilon_{f s}$ & $\begin{array}{l}\text { Energy dissipated in free } \\
\text { space propagation }\end{array}$ & $10 \mathrm{pJ} / \mathrm{bit} / \mathrm{m}^{2}$ \\
\hline$\varepsilon_{m p}$ & $\begin{array}{l}\text { Energy dissipated in } \\
\text { multipath propagation }\end{array}$ & $0.0013 \mathrm{pJ} / \mathrm{bit} / \mathrm{m}^{4}$ \\
\hline $\begin{array}{l}\text { Data Packet } \\
\text { Size }\end{array}$ & Size of a data packet & $500 \mathrm{bytes}$ \\
\hline $\begin{array}{l}\text { Control } \\
\text { Packet Size }\end{array}$ & Size of a control packet & $25 \mathrm{bytes}$ \\
\hline
\end{tabular}

\section{CONCLUSION}

Previous fuzzy logic based clustering protocols are mainly dedicated to handling homogeneous WSNs and do not consider the indegree and outdegree of nodes separately. The proposed protocol developed specifically for a heterogeneous sensor network takes care of the different communication ranges of the heterogeneous nodes and therefore considers the indegree and outdegree of nodes as separate criteria for selection of $\mathrm{CH}$. The proposal takes four input variables for $\mathrm{CH}$ selection - Residual Energy, Indegree, Outdegree and Distance to BS. The proposed protocol has a fuzzy logic which returns two output variables. The same has been considered previously only in DUCF [14] that too for homogeneous WSNs only.

The proposed protocol can be further improved by including other input parameters like coverage, node centrality and other related. At present, the proposed protocol uses multi-hop transmission and assumes the network to be dead when $75 \%$ of the nodes. This can be further refined by checking actual connectivity of the nodes in the network and preparing clusters accordingly. The proposal can be tested with dynamic deployment settings also.

\section{REFERENCES}

[1] W. Dargie and C. Poellabauer, "Fundamentals of wireless sensor networks: theory and practice", John Wiley and Sons. pp. 168-183, 2010.

[2] S. Tarannum, "Energy Conservation Challenges in Wireless Sensor Networks: A Comprehensive Study", Wireless Sensor Network, Vol. 2, pp. 483-491, 2010.

[3] W. R. Heinzelman, A. Chandrakasan, H. Balakrishnan, "Energy-Efficient Communication Protocol for Wireless Microsensor Networks", Proceedings of the 33rd Annual Hawaii International Conference on System Sciences, pp. 10-19, 2000.
[4] O. Younis and S. Fahmy, "HEED: A hybrid, energyefficient, distributed clustering approach for ad-hoc sensor networks", IEEE Transactions on Mobile Computing., Vol. 3, pp. 366-379, 2004.

[5] M. Ye, C .F. Li, G. H. Chen and J. Wu, "EECS: an energy efficient clustering scheme in wireless sensor networks", Proceedings of the 24th IEEE International Performance, Computing, and Communication Conference (IPCCC 2005), pp. 535-540, 2005.

[6] C. F. Li, M. Ye, G. H. Chen and J. Wu, "An energyefficient unequal clustering mechanism for wireless sensor network", Proceedings of the IEEE International Conference on Mobile Adhoc and Sensor Systems Conference, pp. 596-640, 2005.

[7] H. Li, Y. Liu, W. Chen, W. Jia, B. Li, J. Xiong, "COCA: Constructing Optimal clustering architecture to maximize sensor network lifetime", Computers Communications, Vol. 36, pp. 256-268, 2013.

[8] Y. Liao, H. Qi, W. Li, Load-balanced clustering algorithm with distributed self organization for wireless sensor networks, IEEE Sensors Journal, vol. 13 , no. 5, pp. 1498-1506, 2013.

[9] Z. Xu, Y. Yin and J. Wang, "An Density-based Energyefficient Routing Algorithm in Wireless Sensor Networks Using Game Theory," International Journal of Future Generation Communication and Networking, vol. 5, pp. 99-112, 2012.

[10] W. A. Ellatief, O. Younes, H. Ahmed and M. Hadhoud, "Energy Efficient Density-based Clustering Technique for Wireless Sensor Network", Proceedings of the 8th International Conference on Knowledge and Smart Technology (KST), 2016.

[11] I. Gupta, D. Riordan and S. Sampalli, "Cluster-head election using fuzzy logic for wireless sensor networks", Proceedings of the Communication Networks and Services Research Conference, pp. 255-260, 2005.

[12] J. Kim, S. Park, Y. Han and T. Chung, "CHEF: cluster head election mechanism using fuzzy logic in wireless sensor networks", Proceedings of the 10th International Conference on Advanced Communication Technology, pp. 654-659, 2008.

[13] H. Bagci and A.Yazici "An Energy Aware Fuzzy Unequal Clustering Algorithm for Wireless Sensor Networks," Proceedings of IEEE Conference on Fuzzy Systems, pp. 1-8, 2010.

[14] B. Baranidharan and B. Santhi, "DUCF: Distributed load balancing Unequal Clustering in wireless sensor networks using Fuzzy approach", Applied Soft Computing, Vol. 40, pp. 495-506, 2016.

[15] E. H. Mamdani, "Application of fuzzy logic to approximate reasoning using linguistic synthesis", IEEE Transactions on Computers, vol. 26, no. 12, pp. 1182 1191,1977 